\title{
Fe y razón en el campo práctico-político. Un estudio desde la correspondencia de san Agustín con autoridades públicas
}

\author{
Manfred Svensson \\ UNIVERSIDAD DE LOS ANDES
}

\section{Introducción}

Tanto el pensamiento político de san Agustín como su visión de las relaciones entre fe y razón han sido objeto de una cantidad considerable de atención. Sin embargo, no es fácil dar con estudios que busquen expresamente relacionar estas dos preguntas. Así, cuando preguntamos por su visión de la relaciones entre fe y razón, normalmente estamos preguntando por cómo estas se relacionan, por ejemplo, en nuestro conocimiento de la Trinidad o en nuestro conocimiento del origen del mal ${ }^{1}$. Y viceversa, cuando nos ocupamos de su pensamiento político, el acento suele caer en la doctrina de las dos ciudades o en la concepción agustiniana del dominium como consecuencia de la caída, no en las relaciones entre fe y razón. Así, resulta difícil determinar si acaso y en qué medida el modo en que en los escritos políticos está configurada la relación entre fe y razón es coherente con el modo en que se presenta la misma relación en el resto de la obra de Agustín, o si hay en su obra un modo de relacionar fe y razón que sea específico para el campo del saber práctico. Nuestra preocupación es pues aquí doble: analizar cuán rígido es el modelo de fides quaerens intellectum, y ver si acaso se aplica del mismo modo en todos los campos del saber.

Sobre la Trinidad véase sobre todo la carta 120, y sobre el papel de fe y razón en el conocimiento del origen del mal la discusión con Evodio al comienzo del De libero arbitrio. A continuación cito las obras de SAN Agustín conforme a las últimas ediciones de CCL y CSEL y sigo las abreviaciones del Augustinus-Lexikon. 


\section{Fe y razón en general}

Podemos comenzar dirigiendo la mirada a la relación entre fe y razón de modo general ${ }^{2}$. La doctrina que al respecto presenta Agustín en diversos lugares es conocida: debemos partir por la fe, porque así nos vemos purificados, nos volvemos aptos para recibir semillas de la verdad, y una vez que nuestro espíritu esté sano podrá también comprender ${ }^{3}$. Podemos resumir esta posición diciendo que hay una primacía de la comprensión, en cuanto es ella lo buscado, pero hay una primacía temporal de la fe. Es más, no solo de la fe: hay todo tipo de prerrequisitos morales y espirituales del conocimiento. Hay pasajes en que Agustín virtualmente dice no «crede ut intellegas», sino «ama ut intellegas». ${ }^{4}$ Y aunque él no lo dice, podríamos también calificar de agustiniano un precepto como «adora para entender». Pero la verdad es que la fe no es solo un ítem más entre estos distintos prerrequisitos del conocimiento. Pues la necesidad de la primacía temporal de la fe se funda en al menos dos puntos: no solo en que hay cosas que sobrepasan lo que nuestro intelecto por sí solo puede alcanzar, sino también en el hecho de que requerimos una purificación; la fe actúa, por decirlo así, simultáneamente en ambos frentes, pues en cuanto da conocimiento e implica sometimiento a una autoridad es remedio simultáneo para nuestra ignorantia y nuestra difficultas ${ }^{5}$.

Ahora bien, conviene desde un comienzo tener presente que en esta concepción de las relaciones entre fe y razón confluyen elementos bíblicos y elementos filosóficos. Pues si bien es cierto que es a Isaías 7:9 que Agustín apela una y otra vez - «si no creéis no entenderéis»-, la idea de que se deba pasar por una catarsis para alcanzar el conocimiento y para acercarse o asemejarse a Dios no solo es una doctrina platónica, sino una que Agustín reconoce como tal ${ }^{6}$. Es más, una vez que se ha indicado los motivos por los que conviene empezar por la fe, se puede también afirmar -como de hecho lo hace Agustín- que «es la misma razón la que pide que partamos por la fe» ${ }^{7}$ Y así hay también un sentido en el que hay

2 Para una adecuada exposición de estas cuestiones véase F. vAN Fleteren, «Authority and Reason, Faith and Understanding in the Thought of St. Augustine» en Augustinian Studies 4 (1973) 33-71.

3 Véase util. cred. 1, 2; 9, 21 y 14, 31.

4 Así se podría parafrasear las palabras en Io. ev. tr. 96, 4.

5 Lib. arb. III, 18, 52.

6 Civ. XIV, 5.

7 Ep. 120, 3. 
una primacía temporal de la razón: a lo menos en cuanto muestra que debe haber una primacía temporal de la fe. Incluso en su campo normal de aplicación, por tanto, las relaciones fe-razón son en Agustín algo más complejas que lo que permite sospechar el usualmente citado requisito de primacía temporal de la fe. «Para creer comprende mis palabras, para comprender cree en la Palabra de Dios», dice en un sermón ${ }^{8}$.

Ahora bien, como ya indicamos, es común que se limite esta relación entre fe y razón a ciertos tópicos como nuestro conocimiento de Dios. ¿Es correcto este proceder o debemos pensar también en el campo práctico-político como un campo en el que rige el esquema de primacía temporal de la fe? Al respecto no son muchas las tesis explícitas de Agustín. En la cuestión 48 del de diversis quaestionibus, por ejemplo, pregunta por las relaciones entre fe y razón en tres campos distintos. En primer lugar, hay algunas cosas en las que tendremos que conformarnos por siempre con la fe -como son las historias sobre gestas humanas. En otras realidades el asentimiento a las mismas trae consigo de inmediato la comprensiónesto ocurriría en el caso del conocimiento sobre los números. El tercer tipo de objeto de conocimiento que menciona en este pasaje, aquél en el que hay primero fe y luego se llega a la comprensión, son las cosas divinas? Esta tripartición omite pues el campo práctico: nos habla sobre fe y razón en el conocimiento de Dios, de la historia y de los números, no en el conocimiento sobre qué hacer con nuestra vida en la ciudad. Un pasaje algo más revelador se encuentra en el De utilitate credendi, donde Agustín afirma que si bien en todo campo se requiere de purificación previa para el conocimiento, hay diferencias por campo, y «las cosas humanas son más fáciles (promptiores) de conocer que las divinas» ${ }^{10}$. En esta afirmación lo que tenemos es pues una continuidad con el modo en que fe y razón se entienden en el campo de las cosas divinas, pero también un posible reconocimiento de que en este campo, el de las cosas humanas, el requisito de la primacía temporal de la fe se aplica de un modo menos estricto, dada la presunta

\footnotetext{
8 S. $43,9$.
}

9 Div. qu. 48. Credibilium tria sunt genera. Alia sunt quae semper creduntur et numquam intelleguntur, sicut est omnis historia temporalia et humana gesta percurrens; alia quae mox ut creduntur intelleguntur, sicut sunt omnes rationes humanae vel de numeris vel de quibusque disciplinis; tertium quae primo creduntur et postea intelleguntur, qualia sunt ea quae de divinis rebus non possunt intellegi, nisi ab his qui mundo sunt corde, quod fit praeceptis servatis, quae de bene vivendo accipiuntur.

10 Util. cred. 12, 27. 
facilidad para conocer las cosas humanas. Con todo, si queremos conocer algo más de lo que Agustín piensa al respecto, no parece haber más tesis explícitas a analizar, sino que tendremos que analizar su propia práctica al proceder en este campo. Eso es lo que intento a continuación.

\section{El público mixto}

Pero debo entonces explicitar algo más el título del presente artículo. Lo que aquí se revisa no es fe y razón en ciertos tópicos políticos, sino más bien en ciertos escritos políticos de Agustín o, más exactamente, en los escritos - no exclusivamente políticos- dirigidos a políticos, a hombres en servicio público activo o interesados en el mismo. Es decir, mi foco de interés aquí más que un cierto tópico es un cierto público, el modo en que san Agustín coordina fe y razón al tratar con cierto público. Este público - destinatario de obras como La Ciudad de Dios y en parte también del De Trinitate - nos es bastante conocido por algunas cartas, en las cuales me concentraré a continuación. Se trata de los dos siguientes conjuntos: 135-8 y 152-155. El primer grupo es de finales del año 411 o comienzos del 412, mientras que el segundo data del 413 o 414. Es decir, me restrinjo a un período de unos tres años, que nos sitúa en el inicio de la redacción de La Ciudad de Dios, período en que además está iniciándose la controversia con el pelagianismo.

¿Cómo pueden ser caracterizados los interlocutores de Agustín en esta correspondencia? No hay que imaginar un bloque uniforme del paganismo contra un bloque uniforme del cristianismo. En el caso de las cartas 135-8 nos encontramos con Volusiano, un pagano filosóficamente ecléctico ${ }^{11}$, con genuino interés por el cristianismo. Este le escribe a Agustín a través de Marcelino -el funcionario imperial al que está dedicada La Ciudad de Dios- presentándole algunas dudas. Marcelino adjunta una carta propia, en la que advierte a Agustín que cualquier cosa que éste responda a Volusiano será leída por éste a un amplio público. Y dentro de dicho amplio público se encuentran no solo cristianos como Marcelino y paganos sinceramente interesados por el cristianismo, como Volusiano, sino también hombres que adoptan una actitud de desdén: Marcelino cuenta que en las conversaciones ha estado presente un potentísimo terrateniente que en tono irónico adula la sabiduría de

11 Esto se desprende de Ep. 135, 1. 
Agustín mientras se jacta de no haber sido convencido por éste en una conversación que habrían tenido ${ }^{12}$.

En el caso de las cartas 152-155 la situación es distinta. Ahí trata con Macedonio, el vicarius Africae, cabeza de la administración romana en el norte de África ${ }^{13}$. La correspondencia entre Agustín y Macedonio parte con Agustín solicitando clemencia para un condenado a muerte. No está conservada dicha carta, pero sí la primera respuesta de Macedonio, la carta 152. En su respuesta Macedonio por una parte manifiesta preocupación por el modo en que tal impunidad fomentaría la irresponsabilidad, y por otra parte manifiesta dudas respecto de la pertinencia de que el obispo haga tal solicitud: «cuestiono de modo firme -le escribe- que esto provenga de la religión» (vehementer ambigo, utrum istud ex religione descendat) ${ }^{14}$. En la traducción de la Biblioteca de Autores Cristianos esto está traducido como «yo dudo mucho de que esto toque a la religión», y en una reciente contribución sobre esta correspondencia, Robert Dodaro ha dado a esta observación un tono tal vez demasiado contemporáneo, parafraseándola como un llamado de Macedonio a que los obispos se limiten a su propio campo de acción, que sería el religioso ${ }^{15}$. Pero cuesta imaginar a Macedonio trabajando con ese tipo de presupuestos modernos. De lo que duda es de la religiosidad de esta sugerencia en concreto: el problema no es si acaso desde la religión puede hablarse a la política, sino si acaso puede venir de una mente religiosa una aparente solicitud de impunidad como ésta. En mi lectura Macedonio, un laico, estaría criticando al obispo no por ser demasiado religioso en sede equivocada, sino por ser demasiado poco religioso.

Sea esto como fuere, este es el público al que se dirigen tanto las grandes obras de Agustín en este período, como la correspondencia que aquí comentamos. Es un público mixto -como el público de hoy- y que, trátese de interlocutores paganos o cristianos, sacerdotes o laicos, plantea con

12 Ep. 136, 3.

13 Para la información que poseemos sobre estos hombres y su papel en la administración cf. F. Morgenstern, Die Briefpartner des Augustinus von Hippo. Prosopographische, sozial- und ideologiegeschichtliche Untersuchungen (Bochumer historische Studien, Alte Geschichte 11; Bochum 1993).

14 Ep. 152, 2.

15 R. Dodaro, «Augustine and the Possibility of Political Conscience» en C. MAYer (ed.) Augustinus - Ethik und Politik Augustinus-Verlag bei Echter, (Würzburg 2009) 223242, aquí 224. 
coraje sus dudas, sean estas respecto de fundamentos de la fe o respecto de aplicaciones prácticas concretas. ¿Cómo se coordinan ante ese público fe y razón en las respuestas de Agustín?

\section{Correspondencia con Macedonio}

Comienzo atendiendo a la correspondencia con Macedonio. La carta 155 -última entre ambos- parte alabando a Macedonio por su amor a la «república celeste y divina cuyo rey es Cristo». El llamado tiene por claro objeto poner en tensión a su interlocutor, quien en realidad no había dado gran seña de tal amor en la carta 154. Ahí más bien había deslizado una sutil queja porque Roma no salía lo suficientemente bien parada en la obra de Agustín - La Ciudad de Dios- cuyos primeros libros habían recién sido publicados. La queja de Macedonio es la de un amante herido, porque no se ha hablado suficientemente bien de su amada, Roma. Pero «si tu administración -insiste Agustín a Macedonio- consiste en nada más que velar porque los hombres no sufran molestias», «sin referir tal paz a ninguna otra cosa», los esfuerzos no servirán de nada ${ }^{16}$. Esto no parece decirnos nada sobre fe y razón, pero sí me parece que pone un marco, el de la tensión, de la ruptura con el mero bienestar, frecuente en Agustín y apto para introducir su tratamiento de nuestro problema.

Pero lo que aquí nos interesa es ver la forma concreta que este espíritu de tensión toma en algunos de los argumentos presentados por Agustín en esta correspondencia. El primer lugar en el que lo podemos ver es en su respuesta a Macedonio respecto de la legitimidad de la intercesión a favor de un condenado a muerte. La posición de Agustín parece bien sencilla: parece reconocer el derecho de la autoridad a sostener la pena de muerte, pero también el derecho del obispo a pedir clemencia. ¿Pero cómo se fundamentan estos dos derechos? ¿Cómo discute Agustín? En primer lugar, simplemente partiendo por lo concedido por el adversario: «responderé a tus palabras con otras palabras tuyas», comienza diciéndole, «voy a usar los puntos que ya concediste, para con ellos echar abajo la mole que parece impedir mi intercesión $\rangle^{17}$. Y lo que ha concedido Macedonio es que todos los pecados parecen más fáciles de perdonar cuando el reo promete corregirse. Esto suena bastante trivial, y tal vez lo es. Pero lo que Agustín tiene que probar contra Macedonio es efectivamente algo

\footnotetext{
16 Ep. 155, 3, 10.

17 Ep. 153, 1, 2.
} 
muy sencillo: que no hay conflicto entre justicia y misericordia, que se puede pedir misericordia sin detrimento para la justicia.

¿Cómo defiende Agustín dicha posición? Una interpretación recientemente propuesta es la de afirmar que Agustín hace reflexionar a Macedonio poniéndole enfrente dos pasajes bíblicos aparentemente opuestos. Agustín por una parte cita (desde luego con aprobación) Romanos 13, que legitima la decisión de Macedonio al afirmar que la autoridad tiene la espada para castigar como vengador al que hace lo malo ${ }^{18}$, pero por otra parte presenta el relato de la mujer adúltera en Juan 8 , como un caso que nos debe enseñar a ser movidos a misericordia ${ }^{19}$. Agustín no estaría presentando a la autoridad pública un precepto al cual atenerse, sino que le estaría concediendo a Macedonio el punto de este -mediante la cita de Romanos 13- pero llevándolo a ampliar su horizonte, refiriéndolo a otro texto bíblico que también debe ser considerado, obligándolo así a pensar por cuenta propia. Constantemente, en efecto, se encuentra en estas cartas textos que parecen llamar a tal ampliación del horizonte concediendo un punto al interlocutor para a continuación acentuar el otro polo del problema: «Es provechosa tu severidad -le escribe a Macedonio-, por cuyo ministerio se ayuda también a mi propia paz; pero también es provechosa mi intercesión, por cuyo ministerio se templa también tu severidad $»^{20}$. Ampliado su horizonte de este modo, Agustín espera que el gobernante no piense sólo en garantizar la paz temporal.

Coincido con Dodaro, quien propone este tipo de lectura de la carta y que la ve como el más temprano caso de un obispo intentado realizar algo así como un trabajo de «formación de la conciencia» de un político. Pero dado que Dodaro alude a la conciencia, podríamos aquí añadir algo más tomando en consideración el tratado 33 sobre el Evangelio de Juan, que se ocupa de este mismo pasaje, Juan 8 , y en que la conciencia aparece de modo expreso. Este tratado, por cierto, también parece ser del año 414. Ahí Agustín parte por reconstruir la escena de modo tal que los fariseos aparezcan queriendo encerrar a Jesús mediante un dilema moral. Como Jesús se ha ganado la aprobación de la multitud por su mansedumbre y es reconocido como veraz, los fariseos considerarían que la única manera de encerrarlo es plantearle un dilema moral que lo lleve a perder su re-

\footnotetext{
18 Ep. 153, 6, 19.

19 Ep. 153, 4, 9- 4, 11.

20 Ep. 153, 6, 19.
} 
putación de justo: al presentarle a la mujer adúltera, lo obligan o bien a conceder que debe ser apedreada conforme a la ley (con lo cual perdería su reputación de manso) o bien que debe ser liberada contrariamente a lo establecido por Moisés (con lo cual se revelaría como injusto) ${ }^{21}$. Como se puede apreciar, estamos ante el mismo tipo de dilema del que se trata en la carta a Macedonio: el de una aparente alternativa entre la justicia y la misericordia. ¿Cómo sale Cristo del dilema según el tratado 33 de Agustín? La lectura de Agustín es que el dilema es desarmado, que el llamado a que el sin pecado arroje la primera piedra no puede ser leído como opción por uno de los cuernos del dilema. Pues lejos de tratarse de una opción por la mansedumbre en lugar de la justicia, Agustín interpreta las palabras de Jesús como una intensificación de la justicia. Comentando las palabras de Jesús escribe que «esto es de punta a cabo la voz de la justicia» (baec est omnino vox iustitiae). No se opta por uno de los cuernos del dilema, sino que se intensifica uno, el de la justicia, para desde ahí desarmar la trampa. Porque es precisamente la intensificación de la justicia, que se opera al conducir a los acusadores ante el tribunal de su propia conciencia, lo que los lleva a la mansedumbre ${ }^{22}$.

Si tomamos en consideración este texto paralelo, tal vez no sea necesario pensar con Dodaro que Agustín pone en tensión Juan 8 y Romanos 13. Para el propósito que Agustín tiene -llevar a que Macedonio deje de contraponer justicia y mansedumbre- le basta Juan $8^{23}$. En cualquier caso, en este primer caso en el que nos detenemos no hay nada de apelación a argumentos filosóficos, sino que Agustín hace reflexionar a un interlocutor desde el solo texto bíblico.

Dirijo ahora mi atención a la carta 155, parte del mismo intercambio, pero que presenta dos diferencias respecto de la anterior. Por una parte, la discusión se amplía mucho más allá del problema específico que había dado origen a la controversia, para abarcar ahora toda la cuestión de las funciones del gobernante; por otra parte, parece cambiar también la estrategia. Pues ahora Agustín parte de un modo polémico contra la filosofía pagana. Crea la escena hablando de la amistad, la cual solo es verdadera al ser gratuita ${ }^{24}$. Sobre esto, dice, «también dijeron muchas cosas los filóso-

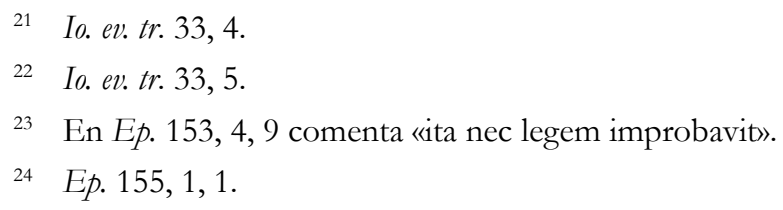


fos, pero entre ellos no hay verdadera piedad $»^{25}$. Estas palabras abren una invectiva sobre todo antiestoica, y lo que nos interesa aquí es ver el tipo de argumentos y autoridades que utiliza en su contra.

En primer lugar, critica que los filósofos habrían intentado «fabricarse la felicidad a su modo». Lo criticado es, desde luego, la pretensión de autosuficiencia. Siguiendo un proceder usual en Agustín, lo que hace no es atacar directamente tal pretensión, sino apuntar al curioso hecho de que sea precisamente la Stoa la que legitima el suicidio. Los mismos que se consideran de una autosuficiencia tal que habrían podido ser felices incluso bajo tortura, son los que han creído que hay situaciones en las que el sabio puede quitarse la vida. De modo que en este caso Agustín no parte apelando a un texto bíblico, ni siquiera intenta refutar al sabio estoico desde fuera del sistema del mismo, sino que apunta a contradicciones intrasistémicas. A continuación, Agustín insiste con el mismo argumento, pero esta vez con base textual, apelando a las páginas finales de las Disputaciones Tusculanas de Cicerón ${ }^{26}$. Todo el peso de la argumentación está al comienzo puesto aquí, en la tensión interna dentro del pensamiento del adversario. Esto se ve luego complementado por una sección en la que Cicerón es puesto contra Cicerón, pues Agustín encuentra un pasaje en el que Cicerón concede que «esta vida en realidad es una muerte de la que me podría lamentar si quisiese», sentencia que Agustín califica como «más sana» que las palabras de las Tusculanas ${ }^{27}$. La discusión de los paganos en esta carta tiene pues dos aristas: por una parte Agustín intenta mostrar lo que le parecen contradicciones internas en el pensamiento de éstos, por otra los cita como testimonio a favor de la verdad que defiende: estamos al comienzo de la controversia antipelagiana, y no es la única vez en ella que Cicerón será citado como testigo de la condición caída del hombre.

Pero en este punto me parece que la carta comienza a dar un giro en cuanto al modo de la exposición. Esto se vuelve patente en la sección 2, 5, que comienza con Agustín justificándose por esta carta que puede resultar una carga muy pesada (si prolixa epistula onerosus sum...). En esa pausa se detiene a reflexionar sobre el hecho de que los filósofos citados son brillantes (acutissima et excellentissima ingenia), pero que por buscar la

\footnotetext{
25 Ep. 155, 1, 2.

26 Ep. 155, 1, 3. La referencia es a las páginas finales de las Disputaciones Tusculanas: V, 38, $110-\mathrm{V}, 41,118$.

27 Ep. 155, 1, 4.
} 
verdad por cuenta propia no la han encontrado $(2,5)$. Con eso se acaba la discusión con los filósofos, y Agustín llama a arrojar lejos las vanidades y locuras de tales hombres $(2,6)$. Pero queda un tercio de la carta, y éste comienza con Agustín dirigiéndose a Macedonio con las siguientes palabras: «Como sé que eres un amante de la república, ve cuán claro se puede ver en las Sagradas Escrituras que la ciudad y el hombre no se vuelven felices a partir de cosas distintas» $(2,7)$. Pasamos pues aquí a una argumentación escritural respecto de un aspecto de la filosofía política. Estas palabras introducen, de hecho, una cita del salmo 143: «Bienaventurado el pueblo cuyo Dios es el Señon». Podríamos aquí parafrasear y explicar diciendo «bienaventurado el pueblo que no pone su bien en el mero bienestar terrenal, porque los pueblos, tal como los hombres, deben estar en tensión hasta que descansen en Dios». Según este salmo son solo «los hijos de los extranjeros» los que afirman que se puede llamar feliz a un pueblo por la sola abundancia de bienes terrenales. Comentando el mismo salmo en el sermón 32 Agustín insiste en que conformarse con felicidad temporal es propio de «alienigenae» ${ }^{28}$. Parece pues que este punto es constantemente reforzado desde un texto bíblico. ¿Se trata entonces de una verdad exclusiva de los cristianos? No. La verdad es que la doctrina en cuestión es moneda mucho más corriente de lo que Agustín quiere conceder aquí. Si bien no para hablar respecto de la felicidad, sino respecto de la justicia, el mismo Agustín trabaja en La Ciudad de Dios con este tipo de paralelos entre individuo y sociedad ${ }^{29}$. Y el origen de tal argumento -con la misma imagen de los individuos en la ciudad como letras en un discurso- no se remonta al salmista, sino a Platón ${ }^{30}$. En el último tercio de la carta nos encontramos pues con Agustín formulando un elemento de teoría política clásica, pero apoyado en un salmo y sin mención del obvio eco clásico de su tesis. Para nosotros esto puede no resultar evidente a primera vista, pero es difícil pensar que sus interlocutores educados no hayan captado dicho eco.

Si mi lectura de la estrategia de Agustín en esta carta es correcta, esto significa que el eje de la argumentación no se ajusta a moldes típicos. No parte por mover a la fe, para desde ahí mover a sus interlocutores a la comprensión; ni parte por la razón, afirmando que paganos y cristianos tienen mucho en común, para tras eso discutir sobre lo que es específico de los cristianos apelando a textos bíblicos. Por lo que hemos podido ver

28 S. 32, 18.

29 Véase Civ. IV, 4, pasaje que incluye material del De Republica de Cicerón.

30 Véase Platón, Politeia II, 368a. 
aquí, su proceder es más bien el inverso: parte por acentuar la antítesis entre paganismo y cristianismo, pero precisamente eso lo hace desde la razón, no desde las Escrituras; luego intenta exponer un punto que es común a cristianos y a lo mejor de la filosofía política clásica -pero esto lo hace solo apelando a un texto bíblico-. Provisionalmente podemos entonces decir que si hay aquí un espíritu de fides quaerens intellectum, es eso: un espiritu y no una regla a la cual se atenga estrictamente; su práctica concreta es mucho más flexible que eso.

\section{Correspondencia con el círculo de Marcelino}

Dirijamos ahora la mirada al conjunto 135-138. Los destinatarios específicos de las cartas son el cristiano Marcelino y el pagano Volusiano, pero están claramente destinadas a lectura pública en un círculo mixto como el que antes hemos descrito. El conjunto de preguntas del círculo es diverso: hay, por ejemplo, dudas respecto de la relación del cristianismo con el Antiguo Testamento: si Dios ofreció un plan nuevo, es porque el antiguo tiene que haber sido malo. Hay también preguntas respecto de la compatibilidad de la religión cristiana con la tradición romana, particularmente con su ética política. Finalmente, hay dudas respecto de los milagros -si acaso el Creador del mundo pudo estar encerrado en el vientre de una mujer por nueve meses-. Pero también esta pregunta por los milagros tiene un ingrediente político-cultural: lo que se busca determinar es si acaso este Jesús es más grande por sus milagros que otros hombres que también han realizado gestas extraordinarias (la referencia es a Apolonio y Apuleyo) $)^{31}$.

No me voy a ocupar aquí del contenido de las respuestas de Agustín, sino que voy a intentar desentrañar algunos aspectos estructurales. Quiero partir por un punto que puede mostrar bien el espíritu en que abre estas discusiones. Hacia el final de la carta 138 responde a quienes se quejan por los tempora christiana y las catástrofes que estos tiempos han traído consigo. La respuesta de Agustín, tan diferenciada como la que ofrecerá en La Ciudad de Dios, no rechaza las quejas como simplemente falsas, sino que rechaza precisamente la generalización que hay en estas acusaciones, generalización que bloquea la discusión: «este tipo de queja indiferenciada (baec generalis conquestio) es calumniosa», sentencia ${ }^{32}$. Agustín no está pues

\footnotetext{
31 Ep. 136, 1.

32 Ep. 138, 3, 16.
} 
embarcándose en un discurso de choque de las civilizaciones; más bien parece invitar a que se le hagan acusaciones específicas, para ver si pueden ser atendidas. ¿Cómo atiende entonces a aquellas que fueron presentadas? Abre su respuesta a Marcelino diciendo que aunque sus amigos paganos «aun se opongan y resistan, hay muchas cosas por las que su entendimiento (sensus) puede ser persuadido -sea mediante una razón más sutil y rica-, o ciertamente mediante una autoridad a la que consideren indigno resistir» ${ }^{33}$. Lo que debemos ver es entonces en qué consiste esta argumentación más rica y cuáles son estas autoridades.

En estas cartas Agustín tiene que defender el hecho de que unas mismas Escrituras contengan dos planes de salvación distintos, y debe además enseñar a leer las Escrituras, para ver exactamente dónde se encuentra el conflicto entre lo enseñado por éstas y la costumbre romana. Respecto de lo primero, nos volvemos a encontrar con un caso en el que parte por la sola argumentación racional, defendiendo el principio general de que lo que está bien hecho también se puede cambiar, como cambian los preceptos que se dan a un adulto en relación a los que se dan a un niño ${ }^{34}$. Tras enumerar ejemplos de ese tipo de cambios -algunos buenos otros menos buenos- estos son apoyados en esta carta en primer lugar por la autoridad de Terencio, quien escribe que puede ser «lícito para uno hacer impunemente lo que a otro no le es lícito hacer: no por diversidad en la materia (res), sino en aquel que obra» ${ }^{35}$. Este tono prosigue por un buen número de párrafos. Así afirma en un momento que hay que distinguir lo bello (pulchrum) de lo conveniente (aptum). Siempre es bello lo que Dios hace en el mundo, pero varía lo que es apto para cada momento. Pero conocemos bien el origen de estos conceptos: Agustín escribió antes de su conversión una obra de pulchro et apto ${ }^{36}$. No la conservamos, pero desde luego es una obra cuyo contenido no era cristiano, sino que se componía, muy probablemente, de variadas fuentes paganas. La validez de la idea de dos pactos es pues defendida en esta carta principalmente con argumentos populares, con filosofía y con autoridades paganas.

\footnotetext{
33 Ep. 138, 1, 1.

34 Ep. 138, 1, 2.

35 Ep. 138, 1, 4. La referencia es al Adelphoe de Terencio. Significativamente, las cuatro veces que este texto es citado en el corpus agustiniano es en contexto de este tipo de escrito: una vez en civ., y dos veces en Ep. 185.

36 Conf. IV, 13, 20.
} 
Pero según hemos visto, Agustín afirma que podría convencer a los amigos paganos de Marcelino no mediante cualesquiera argumentos, sino mediante lo que llama una argumentación más rica (ratio uberior). También podríamos traducir esto como una argumentación más fructífera o más plena. Vale la pena detenerse en esta expresión. El término uberior aparece un número menor de veces en la obra de Agustín, y casi siempre como adjetivo de realidades precisamente ricas, como el amor. Habla en diversos lugares de un amor más pleno o gracias más ricas ${ }^{37}$. En contraste con eso parece muy peculiar su uso aquí: ni siquiera es la razón en cuanto tal, o alguna doctrina, la que es calificada de rica, sino un tipo de argumentación -casi podríamos decir un método- ${ }^{38}$. Contra lo que parece prevenir con tal expresión, es el riesgo de que al apelar a la razón, a marcos comunes de comprensión, en realidad se esté simplemente abandonando al interlocutor a su plana concepción de lo que es la racionalidad, a la racionalidad como lo razonable (en el sentido de Locke). Se trata de convencerlo apelando a una argumentación más rica, que expanda precisamente su concepción de la racionalidad. Veamos cómo se puede ilustrar esto desde dos preguntas distintas.

Un primer caso es el de la compatibilidad del cristianismo con las costumbres de la república -la queja porque el cristianismo no permite devolver mal por mal, lo cual sería incompatible con el oficio militar- $-^{39}$. Lo que aquí hace Agustín es recitar una serie de testimonios de Cicerón y Salustio en los que estos afirman que Roma ha crecido precisamente por «ignorar las injurias recibidas en lugar de vengarlas», o que César había gobernado «sin olvidar nada excepto las injurias» ${ }^{40}$. Tras citar estas palabras, Agustín concluye en los siguientes términos: «Cuando leen estas cosas en sus propios autores, exclaman e irrumpen en aplausos. [...] Pero cuando se lee que es la autoridad divina la que ordena no devolver mal por mal, [...] entonces se acusa a la religión como enemiga de la república» ${ }^{41}$. No se trata de un proceder exclusivo de Agustín. En Tertuliano, por ejemplo, encontramos respecto de otras materias la misma idea de que «estas son cosas que, si las decimos solo nosotros, son consideradas pretensiones fantasiosas, pero si las dicen los filósofos o poetas, valen como cumbre

\footnotetext{
Véase, por ejemplo, s. 260c, 1 y s. 210, 1, 2.

38 En una ocasión aparece para designar doctrina: s. 22, 8.

39 Marcelino presenta la objeción en Ep. 136, 2.

40 Agustín los cita en Ep. 138, 2, 9.

41 Ep. 138, 2, 10.
} 
de la sabiduría y signo de genialidad $»^{42}$. Ahora bien, en el caso de Agustín el argumento tal vez es menos perfecto de lo que parece, pues omite el hecho de que los dos textos romanos que cita hablan expresamente de olvidar o ignorar, no de perdonar; no es lo mismo (como Agustín parece querer que creamos) lo que proponen los romanos (-el perdón como estrategia de dominación-) y la Biblia. Pero lo que me interesa aquí es la forma y función del argumento: se trata de mostrar al pagano que, producto de sus prejuicios sobre el grupo en el que se origina una enseñanza, se había cerrado de un modo ilegítimo a ella. Una vez mostrado eso se le puede mostrar que tal vez bien leída la Biblia no es enemiga de la república.

Y eso es lo que Agustín hace a continuación, enseñar a leerla. Las palabras concretas respecto de las cuales hay discusión son las del sermón del monte: «Si alguien te golpea en la mejilla derecha, ofrécele también la izquierda» (Mt. 5:39). Lo que requiere Agustín es que tales palabras sean leídas de un modo que no se derrumbe el imperio por tomarlas en serio, pero de un modo que tampoco las vacíe de contenido, porque en dicho caso no habría provisto de una razón más rica. Para resolver esta cuestión realiza algo muy similar a lo que lo hemos visto hacer en la correspondencia con Macedonio. Ahí ya vimos a Agustín respondiendo no desde una doctrina, sino desde una escena, la de la mujer adúltera que es perdonada. Aquí Agustín pregunta qué hace Cristo cuando él mismo es golpeado en la mejilla. Y lo que hace no es golpear de vuelta, pero tampoco callar y ofrecer la otra mejilla, sino pedir que se le muestre qué ha dicho mal como para ser golpeado. «Si somos muy literalistas - escribe Agustín- habría que decir que Él mismo no cumplió con su propio precepto, pues no ofreció la otra mejilla» ${ }^{43}$. Así, muestra que el pasaje tiene que ser interpretado, que al interpretarlo se quita el posible absurdo, pero que no se le quita lo que tiene de desafiante: Agustín por supuesto cree que se debe seguir este precepto de un modo que genera un cambio interior -Cristo no solo no golpeó de vuelta, sino que fue modelo de paciencia hasta la muerte-, y dicho cambio interior también acaba produciendo un tipo distinto de sociedad. La carta termina, en efecto, pensando en cómo sería esto: «Que nos den un ejército tal como la doctrina de Cristo pide que sean los soldados; que nos den tales provinciales, tales maridos, tales cónyuges, tales padres, tales hijos, tales señores, tales siervos, tales reyes, tales jueces, tales deudores y

42 Tertuliano, Apologeticum 49, 1.

43 Ep. 138, 2, 13. En 138, 2, 12 ya se queja por lo que ocurriría si atendiéramos a las meras palabras, «si verba adtenderimus»; idem en 138, 2, 14 «si verba intuemur». 
tales recaudadores de tributo como los exige la doctrina cristiana, y que se atrevan a decir que es enemiga de la república» ${ }^{44}$.

El segundo caso de «amplificación de la razón» que debemos revisar se encuentra en la discusión sobre los milagros, contra los cuales ya conocemos las objeciones de Volusiano. Una vez más Agustín parece estar enfrentado a un dilema: o nombra milagros menores -como convertir agua en vino-, pero entonces se le reprocha que algunos paganos también realizan milagros como esos; o bien nombra los milagros mayores -haber nacido de una virgen y resucitado de entre los muertos-, pero esto parece increíble: «Aquí dirán que no pueden creer que eso haya ocurrido» ${ }^{45}$. En lugar de quedarse él encerrado por el dilema, lo devuelve a sus interlocutores: «¿Qué se puede hacer con hombres que desprecian las cosas menores y no quieren creer las mayores?» ${ }^{46}$. Aquí la racionalidad del adversario está ridiculizada, podríamos decir, como mera «razonabilidad», como mero término medio, y Agustín le ofrece como alternativa «la fe que abre la puerta a la comprensión (intellectur), mientras que la increencia (infidelitas) la cierra» ${ }^{47}$.

Con eso estamos de regreso en exactamente el punto de partida de nuestra discusión: esta frase -intellectui fides aditum aperit-es una versión más -de las menos conocidas- del credo ut intellegam, o de las diversas fórmulas con que Agustín expresa la primacía temporal de la fe. Pero tal como hemos visto en otras situaciones ${ }^{48}$, esa misma primacía de la fe es algo que es objeto de discusión racional; y la fe, aunque sea un prerrequisito, no es de esos prerrequisitos que alguna vez puedan ser dejados atrás.

\section{Conclusión}

Estamos en condiciones de hacer algunas evaluaciones finales. En términos muy sencillos, creo que se puede confirmar lo que los parcos testimonios del de utilitate credendi y la q. 48 permitían presumir: fe y razón se relacionan aquí de un modo similar a como se relacionan en otros campos de la obra de Agustín, pues las «cosas humanas» no son radicalmente distintas de las «cosas divinas» (y porque Agustín es un pensador mucho más

\footnotetext{
44 Ep. 138, 2, 15.

45 Ep. 137, 4, 14.

46 Ep. 137, 4, 14.

47 Ep. 137, 5, 15.

48 Ep. 120, 1, 3.
} 
sistemático de lo que imaginamos). Con todo, como también cabía presumir, salta a la vista también la flexibilidad con que opera. Quiere hacer pensar a sus interlocutores -cristianos o paganos-, ponerlos en tensión, hacerlos racionales y no meramente razonables, y para estos efectos usa absolutamente todos los caminos que estén a su disposición: en alguna ocasión los hace pensar solo desde un texto bíblico, en otra les pide que partan por la fe, en otra argumenta desde lo que ellos ya han concedido; en una les habla desde un texto bíblico pero para defender una doctrina filosófica, en otra se da cuenta de que incluso a los que todavía no son cristianos puede tener que introducirlos en la tarea de interpretación del texto bíblico; en ocasiones intenta mostrar las doctrinas de los filósofos como autorrefutantes, y en otras ocasiones los cita en favor de una visión cristiana de la realidad. En síntesis, es alguien que a pesar de darnos una refinada explicación teórica de cómo hemos de entender las múltiples relaciones entre fe y razón, no permite que en la práctica tal explicación se convierta en una camisa de fuerza que lo obligue a seguir siempre un método determinado. Simplemente usa todos sus recursos para poner en tensión al interlocutor.

Resumen: Este artículo se propone analizar qué tan rígido es el modelo de fides quaerens intellectum en el pensamiento de Agustín, y ver si acaso se aplica del mismo modo en todos los campos del saber, con especial atención a lo político y particularmente a lo político en los escritos dirigidos a políticos, a hombres en servicio público activo o interesados en el mismo.

Palabras clave: Agustín, política, fe y razón.

Abstract: This article aims to analyze how rigid the fides quaerens intellectum model is in the thought of Augustine, and to see if it possibly applies in the same manner in all fields of knowledge, with special attention to politics, and particularly to political aspects of the works directed at politicians, men in active public service or those interested in it.

Key words: Augustine, politics, faith and reason. 